\title{
Efeitos do banho logo após o nascimento sobre as adaptações térmica e cardiorrespiratória do recém-nascido a termo
}

\author{
Effects of bathing after birth on term newborn's thermal and cardiorespiratory adaptation
}

\author{
Vania Elisa M. Pugliesi', Alice D’Agostini Deutsch², Marcia de Freitas ${ }^{3}$, Maria Fernanda P.S. Dornaus ${ }^{4}$, Celso Moura Rebello 5
}

\section{RESUMO}

Objetivo: Determinar se o banho dado ao recém-nascido (RN) logo após o nascimento interfere na transição para a vida extrauterina, especialmente nas adaptações cardiorrespiratória e na termorregulação.

Métodos: Estudo retrospectivo realizado por meio de levantamento de prontuários de $\mathrm{RN}$ admitidos em maternidade de São Paulo, entre janeiro e março de 2006. Foram incluídos RN com boletim de Apgar $\geq 8$ no quinto minuto de vida: peso de nascimento $\geq 2500$ g; idade gestacional $\geq 37$ semanas e sem malformações congênitas, divididos em dois grupos: 'banho', banhados na sala de parto, e 'controle', banhados após a terceira hora de vida. Na admissão do RN na unidade neonatal, as seguintes variáveis foram analisadas: temperatura axilar, frequência cardíaca e respiratória, pressão arterial média e saturação de oxigênio. Analisou-se também o tipo de parto, o tempo decorrido entre o parto e a admissão no berçário e a taxa de aleitamento na sala de parto.

Resultados: Incluíram-se 194 RN, 98 no grupo banho e 96 no grupo controle. Em ambos os grupos, a temperatura corpórea na admissão era similar $\left(36,6 \pm 0,4\right.$ e $36,6 \pm 0,3{ }^{\circ} \mathrm{C}$; $p=0,68)$; frequência cardíaca $(143 \pm 13$ e $146 \pm 14$ bpm; $p=0,26)$ e respiratória $(51 \pm 6$ e $51 \pm 9 \mathrm{mov} / \mathrm{min} ; p=0,90)$, pressão arterial média ( $44 \pm 6$ e $47 \pm 9 \mathrm{mmHg} ; p=0,13$ ) e saturação de oxigênio $(98 \pm 2$ e98 $43 \% ; p=0,99)$ foram semelhantes. A taxa de aleitamento materno (91 e $57 \%$; $p<0.001$ ) e o tempo em sala de parto (95 \pm 21 e $79 \pm 29$ minutos, $p<0,001$ ) foram significativamente maiores no grupo banho.

Conclusões: Neste estudo retrospectivo, o banho na sala de parto em RN a termo e saudáveis não interferiu na adaptação cardiorrespiratória e na temperatura à admissão na unidade neonatal.

Palavras-chave: banhos; recém-nascido; salas de parto; monitorização fisiológica; regulação da temperatura corporal.

\section{ABSTRACT}

Objective: To determine the effect of bathing right after birth on newborn's transition to extra-uterine life, mainly regarding thermoregulation and cardiorespiratory adaptation.

Methods: A retrospective comparative study enrolled infants admitted between January and March 2006 in a private tertiary care maternity hospital in the city of São Paulo, Brazil. Inclusion criteria were: Apgar score $\geq 8$ ( $5^{\text {th }}$ minute), birth weight $\geq 2500$ g; gestational age $\geq 37$ weeks, no congenital anomalies. Newborns were divided in two study groups: 'bath' (bath right after birth) and 'control' (bath after the $3^{\text {rd }}$ hour of life). Outcome variables were evaluated at neonatal unit arrival: body temperature, heart and respiratory rate, oxygen saturation, mean arterial pressure. The time interval from birth to admission in the neonatal unit, the rate of breastfeeding in delivery room and mode of delivery were also analyzed.

Results: 194 newborns were included: 98 in the bath and 96 in the control group. Both groups had similar temperature at admission in the neonatal unit $36.6 \pm 0.4$ and $\left.36.6 \pm 0.3^{\circ} \mathrm{C} ; p=0.68\right)$; heart rate $(143 \pm 13$ and $146 \pm 14 \mathrm{bpm}$; $p=0.26)$ respiratory rate $(51 \pm 6$ and $51 \pm 9 \mathrm{mov} / \mathrm{min} ; p=0.90)$ mean blood pressure ( $44 \pm 6$ and $47 \pm 9 \mathrm{mmHg} ; p=0.13$ ) and oxygen saturation ( $98 \pm 2$ and $98 \pm 3 \% ; p=0.99)$ were also similar. The breastfeeding rate $(91 \%$ and $57 \% ; p<0.001)$ as
Instituição: Hospital Israelita Albert Einstein (HIAE), São Paulo, SP, Brasil ${ }^{1}$ Enfermeira graduada pela Universidade Federal de São Paulo (Unifesp); aluna do curso de Pós-graduação lato sensu em Perinatologia do HIAE, São Paulo, SP, Brasil

${ }^{2}$ Doutora em Medicina pela Faculdade de Medicina da Universidade de São Paulo (FMUSP); Coordenadora médica do Departamento Materno-Infantil do HIAE, São Paulo, SP, Brasil

${ }^{3}$ Doutora em Saúde Pública pela Faculdade de Saúde Pública da USP; médica do Departamento Materno-Infantil do HIAE, São Paulo, SP, Brasil ${ }^{4}$ Mestre em Enfermagem obstétrica e neonatal pela Faculdade de Enfermagem da USP; Coordenadora de Enfermagem da Unidade Neonatal do HIAE, São Paulo, SP, Brasil
${ }^{5}$ Doutor em Medicina pela FMUSP; médico do Departamento MaternoInfantil do HIAE, São Paulo, SP, Brasil

Endereço para correspondência:

Celso Moura Rebello

Rua Engenheiro Teixeira Soares, 165

CEP 05505-030 - São Paulo/SP

E-mail: celso.rebello@uol.com.br

Recebido em: 12/2/09

Aprovado em: 12/6/09 
well as the interval from birth to admission in the neonatal unit (95 \pm 21 and $79 \pm 29$ minutes; $p<0.001)$ were higher in the bath group.

Conclusions: In this retrospective study, bathing after birth in the delivery room did not affect thermoregulation and cardiorespiratory adaptation of term newborns.

Key-words: baths; infant, newborn; delivery rooms; monitoring, physiologic; body temperature regulation.

\section{Introdução}

A manutenção da temperatura corpórea é um dos fatores determinantes para o sucesso da adaptação cardiocirculatória e respiratória do recém-nascido $(\mathrm{RN})$ imediatamente após o nascimento. $\mathrm{O}$ protocolo de atendimento ao $\mathrm{RN}$ na sala de reanimação estabelecido pela Academia Americana de Pediatria orienta a manter a temperatura corpórea, enxugando o $\mathrm{RN}$ e envolvendo-o em campos aquecidos sob calor radiante ${ }^{(1)}$. Sob condições habituais de sala de parto $\left(20-25^{\circ} \mathrm{C}\right)$, a temperatura cutânea do $\mathrm{RN}$ cai cerca de $0,3^{\circ} \mathrm{C} / \mathrm{min}$ e a temperatura central diminui cerca de $0,1^{\circ} \mathrm{C} / \mathrm{min}$ no período imediatamente após o parto, resultando em uma queda de 2 a $3^{\circ} \mathrm{C}$ na temperatura central no processo de adaptação à vida extrauterina ${ }^{(2)}$.

Por outro lado, imediatamente após o nascimento, a pele do RN se encontra envolvida por secreções e sangue, com potencial de contaminar tanto profissionais de saúde como familiares $^{(3,4)}$, o que justifica a ideia presente em muitas culturas de administrar o banho imediatamente após o nascimento. De fato, essa rotina está presente em muitas maternidades, exceto para partos de alto risco ou para os recém-nascidos prematuros ${ }^{(5)}$.

Críticas em relação ao banho logo após o nascimento têm sido feitas em decorrência da possibilidade de facilitar a hipotermia, prejudicando a estabilização cardiorrespiratória e térmica da criança ${ }^{(6,7)}$. Isso implicaria a necessidade de encaminhar o RN para uma unidade de risco (Unidade de Cuidados Intensivos ou Semi-intensivos), resultando em efeitos indesejáveis relacionados não apenas ao vínculo mãefilho como também aos custos e ao tempo de internação ${ }^{(8)}$.

Diante desses questionamentos sobre o momento ideal do banho de RN após o nascimento, fez-se a hipótese de que o banho realizado logo após o nascimento resultaria em maior incidência de hipotermia e de desconforto respiratório, resultando em maior taxa de admissão em setor de cuidados intensivos. Este estudo visa a determinar a influência do banho - realizado logo após o nascimento, em sala de parto - na adaptação cardiorrespiratória e na temperatura corporal do RN a termo, no momento da sua admissão no berçário.

\section{Métodos}

O Hospital Israelita Albert Einstein (HIAE) é um hospital privado localizado na zona sul do município de São Paulo. A população assistida no hospital é de alto nível socioeconômico, com atendimento realizado por médicos de clínica privada e todas as mães têm cuidado pré-natal adequado. O hospital realiza em média 3.000 partos por ano, com taxa de prematuridade de $14,6 \%$ e de baixo peso de $10,8 \%$. A taxa de admissão no setor de risco (Unidade de Terapia Intensiva - UTI, e Unidade de Cuidados Intermediários - UCI) é de 15,1\%. A amamentação em sala de parto é incentivada em todos os partos a termo - desde que mãe e $\mathrm{RN}$ estejam em condições clínicas para tal - e ocorre em cerca de $75 \%$ dos partos.

Após a aprovação pelo Comitê de Pesquisa e Ética, foi realizado um estudo descritivo e retrospectivo a partir de dados secundários tipo caso-controle, sendo analisados os prontuários dos recém-nascidos admitidos entre janeiro e março de 2006. No grupo banho, foram incluídos todos os RN submetidos ao banho logo após o nascimento, ainda na sala de parto. No grupo controle foram incluídas as crianças admitidas imediatamente após o nascimento de um RN do grupo banho e que preenchessem os seguintes critérios de inclusão: classificação como termo adequado para a idade gestacional (idade gestacional $\geq 37$ semanas), percentil de peso $\geq 10$ segundo Lubchenko ${ }^{(9)}$, peso de nascimento $\geq 2500$ g, boletim de Apgar $\geq 8$ no quinto minuto de vida e a não-apresentação de intercorrências ao nascer. Em ambos os grupos foram excluídas as gestações gemelares, os $\mathrm{RN}$ com malformações ou que apresentavam desconforto respiratório inicial.

Os dados foram obtidos por meio de levantamento do livro de registro dos partos e por análise dos prontuários digitalizados do Sistema de Informação e Estatística do HIAE. As variáveis analisadas incluíram a idade da mãe, o número de gestações, a paridade e o tipo de parto (vaginal ou cesáreo). Entre as variáveis dos $\mathrm{RN}$, as seguintes foram analisadas: sexo; peso de nascimento; idade gestacional (avaliada pela data da última menstruação, ultrassom obstétrico e método de Capurro $\left.{ }^{(10)}\right)$; classificação de acordo com o peso de nascimento e a idade gestacional, sendo considerados adequados para idade gestacional aqueles com peso ao nascer entre o percentil 10 e 90 na curva de crescimento de Lubchenko ${ }^{(9)}$; boletim de Apgar no quinto minuto; local de destino do RN 
(berçário de normais ou de risco - UTI neonatal ou UCI) e taxa de amamentação na sala de parto. No local de destino foram analisados, imediatamente após a admissão, a temperatura axilar, a frequência cardíaca, a frequência respiratória, a pressão arterial média, a saturação de oxigênio (avaliada por oxímetro de pulso Datascope ${ }^{\circledast}$, Passport 2), o intervalo de tempo entre o parto e a admissão no local de destino e a presença de desconforto respiratório.

$\mathrm{Na}$ sala de parto, os $\mathrm{RN}$ foram submetidos aos procedimentos de rotina, incluindo a reanimação, a identificação e a avaliação pelo médico neonatologista. $\mathrm{O}$ aleitamento materno na primeira hora de vida foi incentivado para todas as puérperas, desde que o $\mathrm{RN}$ apresentasse boas condições clínicas. Para realizar o banho logo após o nascimento (grupo banho), ainda na sala de parto, foi necessária a solicitação por parte dos pais; além disso, o $\mathrm{RN}$ deveria ser a termo e adequado para a idade gestacional, com peso de nascimento $\geq 2500$ g, boletim de Apgar $\geq 8$ no quinto minuto de vida e não ter apresentado intercorrências ao nascer. A temperatura da sala de parto foi mantida ao redor de $26^{\circ} \mathrm{C}$ e a da água entre $36-37^{\circ} \mathrm{C}$ (aferida por termômetro antes da imersão do $\mathrm{RN}$ ). $\mathrm{O}$ banho, com duração média entre cinco e dez minutos, foi realizado pelo pai e auxiliado pela enfermeira, em berço de acrílico com água suficiente para imersão do recém-nascido até o pescoço, de forma a permitir que todo abdome e a parte superior do tórax fossem cobertos pela água. O banho do recém-nascido foi realizado entre 15 e 30 minutos após o nascimento, sem sabão, de forma que os resíduos de sangue, mecônio e secreções fossem removidos e o vernix mantido. A seguir, o RN foi secado completamente, envolto em toalhas e mantido ao lado da mãe em berço aquecido até o término do parto. Após a transferência do bebê para o seu local de destino, foi mantido em observação sob calor radiante até a terceira hora de vida.

No grupo controle (banho realizado após a terceira hora de vida), o recém-nascido recebeu o mesmo atendimento em sala de parto, com exceção do banho. Após a transferência do bebê para o seu local de destino, o RN também permaneceu em observação sob calor radiante até a terceira hora de vida, quando foi realizado o banho pela enfermeira com a mesma técnica e equipamentos descritos acima.

Os dados contínuos foram analisados pelo teste $t$ de Student ou teste Mann-Whitney, de acordo com as premissas de distribuição normal e mesma variância dos grupos analisados. Para dados qualitativos, foi utilizado o teste do qui-quadrado ou teste Exato de Fisher. Foi adotado como nível de significância o valor de $p<0,05$. O tamanho amostral foi calculado para obter uma diferença de temperatura entre os grupos na admissão no berçário da ordem de $0,2^{\circ} \mathrm{C}$, considerando uma distribuição bicaudal, um nível de significância de 0,05 , um poder de teste de 0,8 e um desvio padrão da temperatura da ordem de $0,4{ }^{\circ} \mathrm{C}$, sendo necessários 150 recém-nascidos ( 75 em cada braço do estudo). A análise estatística foi realizada com o programa SigmaStat versão 4.0 (SPSS Inc.).

\section{Resultados}

Foram incluídos 194 recém-nascidos, 98 no grupo banho e 96 no grupo controle. As características demográficas de ambos os grupos são detalhadas na Tabela 1. Os grupos foram comparáveis em relação a: idade materna, paridade, sexo, peso de nascimento e boletim de Apgar de 5 minutos. Observou-se menor incidência de parto cesáreo entre os $\mathrm{RN}$ do grupo banho ( 49 versus $82 \%, p<0,01$ ) e, embora não tenham sido observadas diferenças em relação ao peso de nascimento, os $\mathrm{RN}$ do grupo controle apresentaram uma idade gestacional ligeiramente inferior aos do grupo banho

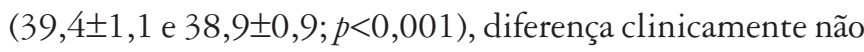
relevante. Foi observado maior tempo transcorrido entre o nascimento e a admissão na unidade neonatal $(95 \pm 27 \mathrm{e}$ $79 \pm 29$ minutos; $p<0,001)$ e maior taxa de amamentação

Tabela 1 - Características demográficas de ambos os grupos

\begin{tabular}{lccc}
\hline & Banho $\mathbf{n = 9 8}$ & Controle $\mathbf{n = 9 6}$ & Valor de $\boldsymbol{p}$ \\
\hline Idade materna (anos) & $32 \pm 4$ & $33 \pm 4$ & 0,592 \\
Primigesta (\%) & 49 & 50 & 0,999 \\
Parto cesárea (\%) & 49 & 82 & $<0,001$ \\
Sexo feminino (\%) & 53 & 51 & 0,573 \\
Peso de nascimento (g) & $3284 \pm 376$ & $3295 \pm 380$ & 0,896 \\
Idade gestacional (semanas) & $39,4 \pm 1,1$ & $38,9 \pm 0,9$ & $<0,001$ \\
Apgar 5 minutos & $9,9 \pm 0,2$ & $9,9 \pm 0,3$ & 0,515 \\
Minutos entre nascimento e admissão na unidade & $95 \pm 27$ & $79 \pm 29$ & $<0,001$ \\
Amamentação na sala de parto (\%) & 91 & 57 & $<0,001$ \\
\hline
\end{tabular}


Tabela 2 - Temperatura e valores cardiorrespiratórios de ambos os grupos no momento da admissão no local de destino

\begin{tabular}{lccc}
\hline & Banho $\mathbf{n = 9 8}$ & Controle $\mathbf{n = 9 6}$ & Valor de $\boldsymbol{p}$ \\
\hline Pressão arterial sistêmica média $(\mathrm{mmHg})$ & $44 \pm 6$ & $47 \pm 9$ & 0,129 \\
Frequência cardíaca $(\mathrm{bpm})$ & $143 \pm 13$ & $146 \pm 14$ & 0,265 \\
Temperatura axilar $\left({ }^{\circ} \mathrm{C}\right)$ & $36,6 \pm 0,4$ & $36,6 \pm 0,3$ & 0,685 \\
Saturação de oxigênio $(\%)$ & $98 \pm 2$ & $98 \pm 3$ & 0,999 \\
Frequência respiratória (mov/min) & $51 \pm 6$ & $51 \pm 9$ & 0,900 \\
\hline
\end{tabular}

na sala de parto entre os $\mathrm{RN}$ do grupo banho (90,8\% versus $56,8 \%, p<0,01)$.

Não foram observadas diferenças entre os dois grupos de estudo em relação aos parâmetros cardiorrespiratórios avaliados (Tabela 2). Todos os $194 \mathrm{RN}$ estudados foram admitidos no berçário de normais, sem desconforto respiratório, não havendo, na amostra estudada, admissões na UTI neonatal ou na UCI.

\section{Discussão}

Em anos anteriores a este estudo, grande número de pais e obstetras manifestava o desejo e banhava os recém-nascidos em sala de parto. Apesar de boas condições ao nascimento, a presença de desconforto respiratório após o banho e a admissão na UTI neonatal surpreendia a equipe multiprofissional. Dessa maneira, no ano de execução deste estudo, foi determinado que o banho fosse realizado apenas nos casos solicitados, sendo implementada a rotina de banho em sala de parto descrita acima.

Este estudo demonstra, pela primeira vez no nosso meio, que o banho realizado logo após o nascimento em recémnascidos saudáveis a termo não interfere na adaptação cardiorrespiratória e na temperatura do $\mathrm{RN}$ à admissão, não aumentando o risco de internação em um setor de risco por insuficiência respiratória. Nossos resultados estão de acordo aos encontrados por Varda $e t a l^{(11)}$, que relataram que o banho logo após o nascimento não afetou a estabilidade térmica de recém-nascidos a termo. Isto se torna particularmente importante porque a capacidade do $\mathrm{RN}$ manter a temperatura corporal determina muitas funções orgânicas, especialmente a obtenção do oxigênio, sendo que os cuidados prestados na recepção do neonato podem, em teoria, afetar seriamente a sua perda calórica ${ }^{(1,2)}$. Sabe-se que os recém-nascidos perdem calor rapidamente durante o primeiro dia de vida quando são banhados, sendo que a diminuição de temperatura pode variar entre 0,1 a $1,1^{\circ} \mathrm{C}^{(6,7,11,12)}$.

Essa preocupação com a instabilidade térmica do recémnascido resultou em uma recomendação da Organização
Mundial da Saúde determinando que o banho do RN deveria ser evitado antes da sexta hora após o nascimento ${ }^{(11)}$. Apesar dessa recomendação, o banho nas primeiras horas de vida é prática padrão em muitas instituições no Brasil devido à preocupação com a possibilidade de transmissão de infecções para os profissionais de saúde e familiares através do contato com sangue e secreções presentes na pele do $\mathrm{RN}$, incluindo, entre outros, os vírus HIV e hepatites B e $\mathrm{C}^{(4)}$.

No presente estudo, não foram observados efeitos do banho sobre a saturação de oxigênio, frequência cardíaca e respiratória e sobre a pressão arterial média no momento da admissão no setor de destino. Resultados semelhantes foram encontrados por Nako et al em $95 \mathrm{RN}$ a termo banhados logo após o nascimento e $92 \mathrm{RN}$ do grupo controle ${ }^{(5)}$. Embora o grupo de $\mathrm{RN}$ submetidos precocemente ao banho registrasse uma temperatura retal em média $0,3^{\circ} \mathrm{C}$ maior do que a do grupo controle, não foram observadas diferenças em relação às frequências cardíaca e respiratória, à pressão arterial média ou em relação à morbidade de ambos os grupos ${ }^{(5)}$.

Nossos resultados demonstram que o banho em sala de parto possibilitou um maior contato do $\mathrm{RN}$ com os pais, identificado pela chegada do $\mathrm{RN}$ ao local de destino cerca de 15 minutos depois do grupo controle. Essa diferença de tempo decorrido para admissão na unidade de destino foi devida, também, a uma maior taxa de amamentação na sala de parto do grupo banho em relação ao grupo controle (91\% versus $57 \%, p<0,001$ ), porém esta diferença de tempo não teve magnitude suficiente para aumentar o risco de desfechos principais desfavoráveis: insuficiência respiratória e menor temperatura axilar no momento da admissão.

Sabe-se que o banho de imersão promove grande conforto ao recém-nascido, como pode ser observado pelo relaxamento, pelos movimentos de mãos e pés dos $\mathrm{RN}$ durante o banho e pelo fato de que eles adquirem um estado sustentado de alerta, tornando-se menos agitados após o procedimento ${ }^{(13)}$. De fato, admite-se que o banho de imersão é uma situação que propicia uma série de trocas e ajustes interacionais entre o adulto e o recém-nascido, proporcionado pela estimulação tátil com os pais e outros cuidadores ${ }^{(14,15)}$. Esses fatores podem 
estar associados à maior adesão à amamentação na primeira hora de vida entre os RN do grupo banho.

Uma possível explicação para os nossos achados é que a diferença entre a temperatura corporal do $\mathrm{RN}$ e do meio externo observada após o nascimento poderia ter um papel fisiológico na adaptação à vida extrauterina, desde que mantida em certos limites. A significativa perda calórica por dissipação para o meio ambiente resultaria no resfriamento da pele (cuja temperatura no ambiente intrauterino é $0,5^{\circ} \mathrm{C}$ superior à materna), com o consequente estímulo das terminações nervosas cutâneas, havendo ativação do centro respiratório e provocando vasoconstrição, o que resulta em aumento da resistência vascular periférica e auxilia na adaptação circulatória ${ }^{(16,17)}$. Sob esse prisma, o banho logo após o nascimento poderia atrapalhar a adaptação pós-natal devido à vasodilatação periférica, levando à hipotensão e relativa hipertensão pulmonar. Ademais, o início da respiração pode ser mais demorado em bebês que nascem diretamente em água quente, situação em que ocorre mínima perda inicial de temperatura corpórea ${ }^{(18)}$. Entretanto, os resultados do presente estudo não demostraram efeitos negativos do banho em sala de parto na adaptação inicial do recém-nascido.

Um fator teórico de confusão em relação à morbidade respiratória seria a maior incidência de parto cesáreo nos recémnascidos do grupo controle, uma vez que foi demonstrada maior morbidade respiratória relacionada ao parto cesáreo, particularmente na ausência de trabalho de parto ${ }^{(19)}$. No entanto, apesar da maior incidência de parto cesáreo entre os $\mathrm{RN}$ desse grupo, não houve correspondente aumento da morbidade respiratória, mesmo imediatamente após o nascimento (o que contraindicaria o banho), embora o presente estudo não conte com dados sobre as indicações das cesáreas ou tempo de trabalho de parto.

Os resultados aqui apresentados demonstram que tanto os nascidos de parto normal quanto de parto cesáreo mantiveram sua temperatura, frequência cardíaca, frequência respiratória e

\section{Referências bibliográficas}

1. American Academy of Pediatrics, AAP. Neonatal resuscitation program international activity report. AAP National Conference and Exhibition; 2002 Oct 18; Boston, USA. Elk Grove Village, IL: AAP; 2002.

2. Stoll BJ. The newborn infant. In: Kliegman RM, Behrman RE, Jenson HB, Stanton BF, editors. Nelson textbook of pediatrics. $18^{\text {th }}$ ed. New York: Elsevier; 2007. p. 433-40.

3. Lund $\mathrm{CH}$, Osborne JW, Kuller J, Lane AT, Lott JW, Raines DA. Neonatal skin care: clinical outcomes of the AWHONN/NANN evidence-based clinical practice guideline. J Obstet Gynecol Neonatal Nurs 2001;30:41-51. oximetria de pulso adequadas após o banho em sala de parto. O desenho do estudo adotado apresenta limitações que devem ser levadas em consideração. Por se tratar de uma análise retrospectiva, não foi possível realizar uma randomização dos recém-nascidos nos dois grupos. Embora o número de recémnascidos estudados tenha sido superior ao cálculo do tamanho amostral, o desenho de estudo adotado deve ser levado em consideração na análise global dos resultados. Sem dúvida, as conclusões finais seriam mais contundentes se o estudo fosse conduzido de forma prospectiva, randomizada e controlada.

Apesar dessas considerações, a presente pesquisa forneceu evidências para mudar a rotina dos cuidados de enfermagem na unidade neonatal do HIAE. Atualmente, o banho de imersão é feito no momento da admissão no berçário em todos os RN estáveis que não vivenciaram essa prática na sala de parto, em contraste com a rotina anterior, em que o banho era realizado apenas depois de três ou quatro horas de vida. O tempo de observação do $\mathrm{RN}$ foi reduzido de três para apenas uma hora dentro da unidade neonatal, favorecendo ainda mais a amamentação e os vínculos familiares. A flexibilidade do momento do banho é recomendada, de acordo com a estabilidade do RN e o desejo da família. O primeiro contato dos pais com seu bebê é significativo no processo de apego. $\mathrm{O}$ banho favorece uma interação harmoniosa com os pais, além de propiciar estimulação tátil e auxiliar na organização do $\mathrm{RN}^{(20)}$. Esse contato agradável parece influenciar o estado de alerta e a disposição do $\mathrm{RN}$ para mamar na sala de parto.

Pode-se concluir que os resultados deste estudo retrospectivo sugerem que o banho realizado imediatamente após o parto, com controle adequado de sua duração, da temperatura da água e da sala no centro cirúrgico, visando ao relaxamento e à retirada das secreções e sangue, não interferiu nas condições cardiorrespiratórias e na temperatura do RN na admissão no berçário. As mães que optaram pelo banho imediato amamentaram mais seus recém-nascidos logo após o nascimento.
4. Penny-MacGillivray T. A newborn's first bath: when? J Obstet Gynecol Neonatal Nurs 1996;25:481-7.

5. Nako Y, Harigaya A, Tomomasa T, Morikawa A, Amada M, Kijima C et al. Effects of bathing immediately after birth on early neonatal adaptation and morbidity: a prospective randomized comparative study. Pediatr Int 2000;42:517-22.

6. Dahm LS, James LS. Newborn temperature and calculated heat loss in the delivery room. Pediatrics 1972;49:504-13.

7. Raman V, Rekha S, Chandrasekhara MK. Effect of bathing on temperature of normal neonates. Indian Pediatr 1996;33:340. 
8. Behring A, Vezeau TM, Fink R. Timing of newborn first bath: a replication. Neonatal Netw 2003;22:39-46.

9. Lubchenco LO, Hansman C, Dressler M, Boyd E. Intrauterine growth as estimated from liveborn birth-weight data at 24 to 42 weeks of gestation. Pediatrics. 1963;32:793-800.

10. Capurro H, Konichezky S, Fonseca D, Caldeyro-Barcia R. A simplified method for diagnosis of gestational age in the newborn infant. J. Pediatr 1978;93:120-2.

11. Varda KE, Behnke RS. The effect of timing of initial bath on newborn's temperature. J Obstet Gynecol Neonatal Nurs 2000;29:27-32.

12. Medves JM, O'Brien B. The effect of bather and location of first bath on maintaining thermal stability in newborns. J Obstet Gynecol Neonatal Nurs 2004;33:175-82.

13. Anderson GC, Lane AE, Chang HP. Axillary temperature in transitional newborn infants before and after tub bath. Appl Nurs Res 1995;8:123-8.

14. Darmstadt GL, Dinulos JJ. Neonatal skin care. Pediatr Clin North Am 2000;47:757-82.
15. Gasparetto S, Bussab VSR. Padrões e estados comportamentais de recém-nascidos durante o banho em maternidade: possibilidades de regulamentação e trocas sociais. Rev Bras Cresc Desenv Hum 2000;10: 30-48.

16. Sinclair JC. Management of the thermal environment. In: Sinclair JC, Bracken $M B$, editors. Effective care of the newborn infant. Oxford: Oxford University Press; 1992. p. 40-58.

17. Gandy GM, Adamsons K Jr, Cunningham N, Silverman WA, James LS. Thermal environment and acid-base homeostasis in human infants during the first few hours of life. J Clin Invest 1964;43:751-8.

18. Purves MJ. Onset of respiration at birth. Arch Dis Child 1974; 49:333-43.

19. Dudell GG, Jain L. Hypoxic respiratory failure in the late preterm infant. Clin Perinatol 2006; 33:803-30.

20. Pillegi MC, Policastro A, Abramovici S, Cordioli E, Deutsch AD. Breastfeeding in the first hour of life and modern technology: prevalence and limiting factor. Einstein 2008;6:467-72. 